\title{
Microscopic origins of charge transport in triphenylene systems
}

\author{
Ian R. Thompson, Mary K. Coe, and Alison B. Walker \\ Department of Physics, University of Bath, Bath BA2 7AY, United Kingdom \\ Matteo Ricci, Otello M. Roscioni, and Claudio Zannoni \\ Dipartimento di Chimica Industriale "Toso Montanari” and INSTM, Università di Bologna, Viale del Risorgimento 4, 40136 Bologna, Italy
}

(Received 24 August 2017; revised manuscript received 21 February 2018; published 5 June 2018)

\begin{abstract}
We study the effects of molecular ordering on charge transport at the mesoscale level in a layer of $\approx 9000$ hexa-octyl-thio-triphenylene discotic mesogens with dimensions of $\approx 20 \times 20 \times 60 \mathrm{~nm}^{3}$. Ordered (columnar) and disordered isotropic morphologies are obtained from a combination of atomistic and coarse-grained moleculardynamics simulations. Electronic structure codes are used to find charge hopping rates at the microscopic level. Energetic disorder is included through the Thole model. Kinetic Monte Carlo simulations then predict charge mobilities. We reproduce the large increase in mobility in going from an isotropic to a columnar morphology. To understand how these mobilities depend on the morphology and hopping rates, we employ graph theory to analyze charge trajectories by representing the film as a charge-transport network. This approach allows us to identify spatial correlations of molecule pairs with high transfer rates. These pairs must be linked to ensure good transport characteristics or may otherwise act as traps. Our analysis is straightforward to implement and will be a useful tool in linking materials to device performance, for example, to investigate the influence of local inhomogeneities in the current density. Our mobility-field curves show an increasing mobility with field, as would be expected for an organic semiconductor.
\end{abstract}

DOI: 10.1103/PhysRevMaterials.2.064601

\section{INTRODUCTION}

Displays for smartphones, colored light sources, off-grid solar cells, and curved television screens are examples of applications of organic electronics. Organic semiconductors (OSCs) offer reduced production costs, versatility of synthesis processes, and compatibility with a vast range of substrates including transparent glass, metals, and flexible polymeric material. However, they cannot compete yet with their inorganic counterparts in terms of charge-transport performance [1]. Charge carriers are localized to molecules or conjugated segments of molecules, in the case of polymers, and charge transport is by donor-acceptor hopping [2] rather than by a band mechanism. At room temperature, charge mobility is highly sensitive to molecular packing arrangements due to the short range of electronic orbital overlaps and their strong dependence on the relative orientation and separation of donor-acceptor pairs [3]. Detailed knowledge of material morphologies, such as those illustrated in Fig. 1 for discotic OSCs [4-11], is thus essential for a proper understanding and prediction of charge mobility.

Multiscale simulations that combine models of morphology and charge transport are an important means of optimization of materials and devices for OSC technology, particularly for the screening of candidate materials [12-14]. While the charge mobilities can be measured experimentally [15], synthesizing and characterizing the films is time-consuming and costly. Knowledge of local charge densities and conductive pathways can be critical to understanding heat dissipation and degradation in OSCs [12]. These effects are not captured by the coplanar dimer model commonly used for prescreening organic molecules [16]. Local current density variations can cause self-heating that in turn may lead to higher local conductivity and thereby creates more current flow, leading to a positive feedback loop [17]. However, variations in charge dynamics at atomistic length scales are hard to study experimentally. Localized current flow can be studied by the cumbersome process of introducing an emissive interlayer into the material and measuring the light emitted [18]. This procedure has a resolution of $2-3 \mathrm{~nm}$ and introduces errors due to interfacial interactions with the emissive layer.

A common method to describe charge mobilities is to use the Gaussian disorder model (GDM) [19,20]. This approach assumes a spatially random distribution of sites where the localization length, $a$, is the relevant length scale compared to site separations [21,22]. Using a lattice model to describe transport introduces an error if the lattice parameter is far from the localization length [23].

We investigate the charge-transport properties of hexaoctyl-thio-triphenylene (HOTT, or 8H-TT) molecules whose chemical structure and coarse-grained shape are shown in Fig. 1. From Fig. 1(a) we see that HOTT is a planar molecule, consisting of three benzene rings surrounding a central benzenic ring. The lower panels come from a preliminary atomistic simulation of HOTT used for parametrization of the coarsegrained model. The semiconducting properties of discotic molecules in OSC devices have been studied both theoretically and experimentally [24]. The high aspect ratio of discotic molecules allows us to examine the role of shape anisotropy and structure in charge-transport processes [7]. Systems of these molecules possess a columnar phase and a disordered phase [5-7,25-29]. The drastic change in structure across the phase transition means that it would be impossible to describe 


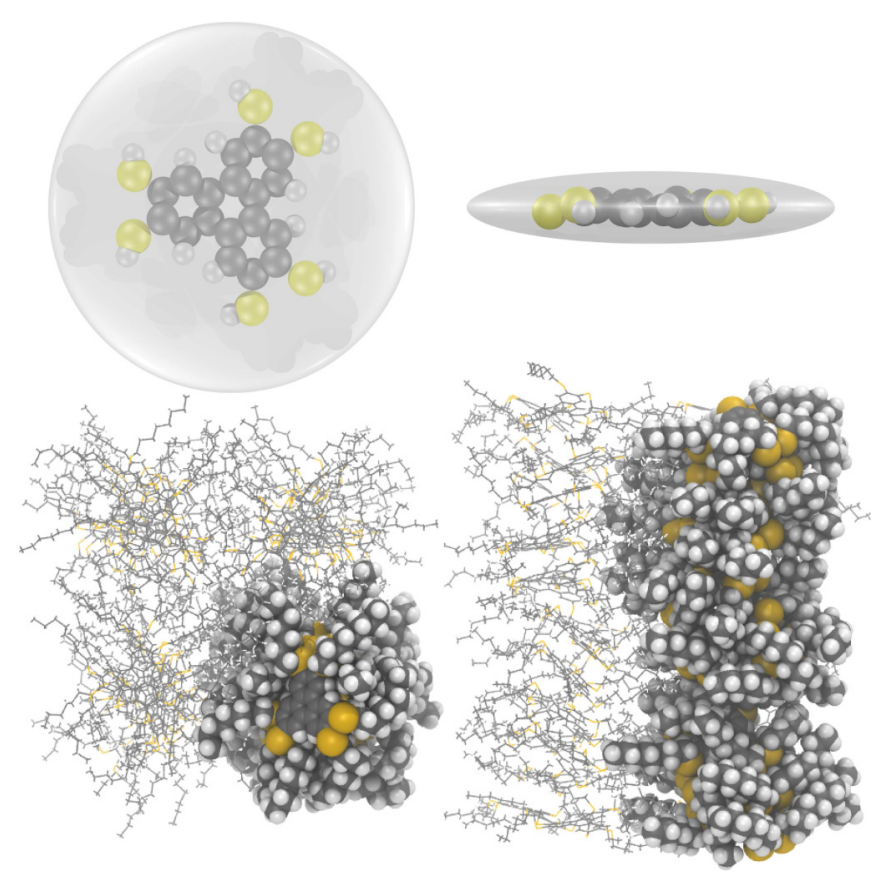

FIG. 1. Top panels: top (left) and side (right) views of the 2,3,6,7,10,11-hexahexyl thiotriphenylene (HHTT) molecule $(R=$ octyl) corresponding to the central core of HOTT with the chains replaced by hydrogen. The oblate Gay-Berne ellipsoid for coarsegrained simulations is also shown. The radius of an HHTT molecule in the $x$ - $y$ plane, $r_{\perp} \approx 0.653 \mathrm{~nm}$, and radial height, $r_{\|} \approx 0.172 \mathrm{~nm}$. Bottom panels: views along (left) and across (right) the column axis direction from atomistic packing simulations.

both phase systems using the same lattice model. Experimental studies [30] have shown that the mobility suddenly increases as the system goes from isotropic to columnar to crystal phases, while within a given phase it gradually decreases with temperature. Discotics possess a strongly anisotropic charge mobility in their columnar phase, much larger along the column axis than perpendicular to it $[9,31,32]$. We compare charge transport in the columnar phase at $T=280 \mathrm{~K}$ and in the disordered phase at $T=400 \mathrm{~K}$.

We have chosen to study HOTT, as a typical discotic molecule, due to the existence of previous studies that we can compare to and build upon. Lamarra et al. [9] investigated 4000 discotic molecules with a simple Miller-Abrahams description of electronic coupling that depends only on molecule separation. Studies on $\sim 1000$ discotic molecules, where charge dynamics were described by a master equation with chargetransfer rates obtained from the Marcus expression and transfer integrals from quantum chemical calculations, showed that structural anisotropy leads to anisotropic charge transport [8]. Rühle et al. demonstrated a similar approach to our own [33] in a cell of 512 molecules, where they neglected metallic contacts. Because their system size is small, they found a single charge-transfer channel that percolated across their entire system. These studies demonstrated the strong dependence of charge mobility upon structural ordering and the possibility of charge percolation pathways, however they did not explore the underlying causes. Herein we try to quantify the difference in transport between the two phases in terms of the microscopic processes and structure within.

We present a mesoscale model of charge transport in a film of $\sim 9000$ molecules, modeling the equivalent of $\sim 700000$ atoms, starting from first principles. This simulation size produces a relative error in the mobility due to finite-size effects of less than 5\% [34,35]. It has been shown elsewhere that a fully atomistic or a united atom molecular-dynamics approach can successfully predict morphologies and transition temperatures with the accuracy of a few degrees for cyanobiphenyls [36-38] and quinquephenyl [39]. However, these atomistic simulations are not feasible at the mesoscale system sizes that we require here, which are of the order of tens of nanometers [40]. For morphologies derived from microscopic calculations, we thus make use of coarse-grained (CG) molecular dynamics (MD) $[41,42]$ parametrized with the help of small-scale atomistic simulations, as will be described in the next section.

Our methods produce a resolution at the microscopic level by describing the charge transport between every pair of molecules as discrete events in time so that we can identify which structural properties lead to varying local current densities. In systems this large, with so many connected pairs, graph theory [43] is a useful tool to analyze the simulated chargetransport trajectories. This approach is an improvement over trajectory plots in that it allows us to investigate correlations between structural and dynamic properties, e.g., the electronic coupling and the observed carrier transport between molecules.

Network analysis has been used to study organic chargetransport networks and kinetic Monte Carlo (KMC) methods before. Jackson et al. used dynamic network techniques to study how the charge-transport network changes over time as the molecules move [44], however they did not model the resultant charge-transport properties. Cottaar et al. used percolation theory on a $2 \mathrm{D}$ lattice to describe the effects of correlated and uncorrelated energetic disorder [45]. They considered the percolative pathways in terms of the current density between lattice sites forming edges. The above study was lattice-based, and although they showed the existence of favored charge pathways, the authors could not relate this to any structural properties of the system, and the energetic disorder was drawn from a chosen distribution. Graph theoretical approaches were also applied to KMC simulations of chemical kinetics by Stamatakis and Vlachos [46]. This is still a system of discrete events, and the underlying algorithm is similar. Indeed, the use of graph theory to describe networks of first-order events is well established in many contexts regarding kinetics. Uniquely we take advantage of the one-to-one spatial equivalence of the transport network with the morphology to quantify the microscopic contributions to macroscopic transport and describe the origins of the phenomena observed.

While other studies have combined the use of CG MD and $\mathrm{KMC}$ to describe transport in molecular systems, they have not been able to combine the system sizes and degree of detail that we present. Furthermore, we will use the microscopic resolution of our model to describe the nature of observed charge transport in terms of the relationship between the difference in site energies and transfer integrals. We explain the prevalence of a rattling motion in OSCs due to the weak effect of the applied field. In this manner, we go further than measuring the charge mobility. To enable truly predictive 
modeling, one must understand the origin of the observed transport phenomena.

In this paper, we present a methodology that allows us to describe charge transport in disordered OSCs with microscopically resolved molecular packings, transport parameters, and charge dynamics. We do not have to resort to tuning parameters, such as reorganization energies or coupling length scales, and our model can describe systems with multiple molecular species, anisotropic molecules, and structural features on all length scales. Below, we describe the CG MD and chargetransport models in Sec. II. We relate the microscopic properties of the system and the resultant charge transport through the use of a directed graph representation in Sec. III. In this way, we identify charge-transport pathways on a microscopic scale, describe their structural features, and measure their spatial extent and separation.

\section{MODELS}

\section{A. Coarse-grained molecular dynamics}

Molecular dynamics can provide a realistic structure of an OSC system for given thermodynamic parameters, provided there is an accurate description of the effective interparticle potential interactions. Force fields can be developed that are suitable for a wide range of temperatures, pressures, and densities and can reproduce multiple phases of matter. In our CG model, we tuned the force field for HOTT on the basis of MD simulations for a fully atomistic model of the molecular system [27]. CGMD has already been shown to reproduce the phase diagram in columnar triphenylenes [27,47]. The CG potential employed for our MD simulations is based on the Gay-Berne (GB) potential, where anisotropic particles are described as rigid bodies of ellipsoidal shape [9,27]. The potential can be considered as a generalization of the LennardJones 6-12 potential, where shape and interaction anisotropies have been introduced.

The GB interaction potential between two particles, $i$ and $j$, therefore depends on their orientations, defined by the unit vectors $\hat{\vec{u}}_{i}, \hat{\vec{u}}_{j}$, and by their center-center separating vector, $\vec{r}_{i j}$ :

$$
\begin{aligned}
U\left(\hat{\vec{u}}_{i}, \hat{\vec{u}}_{j}, \hat{\vec{r}}_{i j}\right)= & 4 \epsilon_{0} \epsilon\left(\hat{\vec{u}}_{i}, \hat{\vec{u}}_{j}, \hat{\vec{r}}_{i j}\right) \\
& \times\left[\left(\frac{\sigma_{0}}{r_{i j}-\sigma\left(\hat{\vec{u}}_{i}, \hat{\vec{u}}_{j}, \hat{\vec{r}}_{i j}\right)+\sigma_{0}}\right)^{12}\right. \\
& \left.-\left(\frac{\sigma_{0}}{r_{i j}-\sigma\left(\hat{\vec{u}}_{i}, \hat{\vec{u}}_{j}, \hat{\vec{r}}_{i j}\right)+\sigma_{0}}\right)^{6}\right],
\end{aligned}
$$

where $\sigma_{0}\left(\epsilon_{0}\right)$ fixes the scales of length (energy), while $\sigma\left(\hat{\vec{u}}_{i}, \hat{\vec{u}}_{j}, \hat{\vec{r}}_{i j}\right)$ and $\epsilon\left(\hat{\vec{u}}_{i}, \hat{\vec{u}}_{j}, \hat{\vec{r}}_{i j}\right)$ correspond to the anisotropic contact distance and potential well depth, respectively. More specifically, the contact distance is

$$
\begin{aligned}
\sigma\left(\hat{\vec{u}}_{i}, \hat{\vec{u}}_{j}, \hat{\vec{r}}\right)= & \sigma_{0}\left\{1-\frac{\chi}{2}\left[\frac{\left(\hat{\vec{u}}_{i} \cdot \hat{\vec{r}}+\hat{\vec{u}}_{j} \cdot \hat{\vec{r}}\right)^{2}}{1+\chi\left(\hat{\vec{u}}_{i} \cdot \hat{\vec{u}}_{j}\right)}\right.\right. \\
& \left.\left.+\frac{\left(\hat{\vec{u}}_{i} \cdot \hat{\vec{r}}-\hat{\vec{u}}_{j} \cdot \hat{\vec{r}}\right)^{2}}{1-\chi\left(\hat{\vec{u}}_{i} \cdot \hat{\vec{u}}_{j}\right)}\right]\right\}^{-1 / 2},
\end{aligned}
$$

TABLE I. Parameters linked to MD, vOTCA, and KMC calculations. The notation is defined in the text for this section.

Gay-Berne parameters [27]

$\mu_{\mathrm{GB}}=1, \chi_{\mathrm{GB}}=0.1948, \chi_{\mathrm{GB}}^{\prime}=0.15$, Eq. (7)

$\sigma_{0}=0.375 \mathrm{~nm}, \epsilon_{0}=1.5897 \mathrm{kcal} / \mathrm{mol}$, Eq. (1)

Parameters deduced from VOTCA calculations

$\lambda=0.43 \mathrm{eV}, \sigma_{\text {col }}=0.064 \mathrm{eV}, \sigma_{\text {dis }}=0.105 \mathrm{eV}$, Eq. (8)

Parameters needed for running KMC

$r_{c}=2.5 \mathrm{~nm}, \Delta t=5 \times 10^{3} \mathrm{ps}, \tau_{\max }=10^{8} \mathrm{ps}, V_{\mathrm{bias}}=2 \mathrm{~V}$, $\epsilon_{r}=2.6[55]$

where

$$
\chi=\frac{k_{\mathrm{GB}}^{2}-1}{k_{\mathrm{GB}}^{2}+1}
$$

is the shape anisotropy parameter, defined by the aspect ratio, $k_{\mathrm{GB}}$. The well depth $\epsilon\left(\hat{\vec{u}}_{i}, \hat{\vec{u}}_{j}, \hat{\vec{r}}\right)$ is determined by the product of two functions:

$$
\epsilon\left(\hat{\vec{u}}_{i}, \hat{\vec{u}}_{j}, \hat{\vec{r}}\right)=\left[\epsilon_{1}\left(\hat{\vec{u}}_{i}, \hat{\vec{u}}_{j}, \hat{\vec{r}}\right)\right]_{\mathrm{GB}}^{\mu}\left[\epsilon_{2}\left(\hat{\vec{u}}_{i}, \hat{\vec{u}}_{j}\right)\right]_{\mathrm{GB}}^{v},
$$

where

$$
\begin{gathered}
\epsilon_{1}=1-\frac{\chi^{\prime}}{2}\left[\frac{\left(\hat{\vec{u}}_{i} \cdot \hat{\vec{r}}+\hat{\vec{u}}_{j} \cdot \hat{\vec{r}}\right)^{2}}{1+\chi^{\prime}\left(\hat{\vec{u}}_{i} \cdot \hat{\vec{u}}_{j}\right)}+\frac{\left(\hat{\vec{u}}_{i} \cdot \hat{\vec{r}}-\hat{\vec{u}}_{j} \cdot \hat{\vec{r}}\right)^{2}}{1-\chi^{\prime}\left(\hat{\vec{u}}_{i} \cdot \hat{\vec{u}}_{j}\right)}\right], \\
\epsilon_{2}=\left[1-\chi^{2}\left(\hat{\vec{u}}_{i}, \hat{\vec{u}}_{j}\right)^{2}\right]^{-1 / 2},
\end{gathered}
$$

while the parameter

$$
\chi^{\prime}=\frac{\left(k_{\mathrm{GB}}^{\prime}\right)^{1 / \mu_{\mathrm{GB}}}-1}{\left(k_{\mathrm{GB}}^{\prime}\right)^{1 / \mu_{\mathrm{GB}}}+1}
$$

is defined in terms of the well depth anisotropy $k_{\mathrm{GB}}^{\prime}$, i.e., the ratio between well depths for the side-by-side and end-to-end interactions, respectively. The form of the GB potential can be adjusted to a specific molecule by varying the parameters $\mu_{\mathrm{GB}}, v_{\mathrm{GB}}, k_{\mathrm{GB}}, k_{\mathrm{GB}}^{\prime}$, with the exponents $\mu_{\mathrm{GB}}, v_{\mathrm{GB}}$ tuning the orientational dependence of the energy. For $k_{\mathrm{GB}}$ and $k_{\mathrm{GB}}^{\prime}$ values smaller than unity, the potential describes disklike mesogens (oblate ellipsoids) and their discotic and columnar mesophases [27,48]. The Gay-Berne parameters obtained from microscopic level simulations in [27] are shown in Table I.

\section{B. Charge-transport model}

\section{Kinetic Monte Carlo simulations}

We took roughly equally sized samples from the $\mathrm{CG}$ morphologies, and they were replicated periodically in the $x$ and $y$ directions while metallic boundary conditions were applied in the $z$ direction to model electrodes. Axes with unit vectors $\vec{e}_{x}, \vec{e}_{y}, \vec{e}_{z}$ were defined as shown in Fig. 3 .

Charge motion is calculated using kinetic Monte Carlo (KMC) methods [49] as it allows for interactions between the charge carriers and describes all mechanisms on a realistic timescale. Hopping rates are determined by Marcus theory where transfer integrals, representing electronic coupling, and hopping site energies are derived from electronic structure and electrostatic interactions between the charge carriers. The 
transfer integrals are highly sensitive to the relative orientations of the orbitals and the hopping distances involved in the transfer process [3] and thus on the local packing. The parameters determining packing and charge hopping rates are determined from electronic structure calculations, and there is no need for parameters to be fitted to experiment. This feature of off-lattice KMC means that one can quantitatively compare charge transport properties predicted by our model across many different materials and morphologies.

We began by defining the center of mass for each molecule as a possible charge hopping site, with a maximum occupancy of one free charge carrier. We used Marcus hopping rates [50] to describe the hopping:

$$
\kappa_{i j}=\frac{\left|J_{i j}\right|^{2}}{\hbar} \sqrt{\frac{\pi}{\lambda k_{B} T}} \exp \left[-\frac{\left(\Delta G_{i j}+\lambda\right)^{2}}{4 \lambda k_{B} T}\right],
$$

where the total reorganization energy, $\lambda=\lambda_{\text {inner }}+\lambda_{\text {outer }}$, was calculated for charge transport between HOTT molecules, the same value used for all molecule pairs in the system. There are two major contributions to the energy of free charges in the system: the molecular orbital energy of the host molecule, and the electrostatic energy of other free charges and any externally applied electric field across the system. We calculated the highest occupied molecular orbital (HOMO) and lowest unoccupied molecular orbital (LUMO) energy levels for each molecule allowing for polarization of neighboring molecules using the Thole multipole model [51]. We calculated the transfer integral, $J_{i} j$, for pairs of molecules with a separation, $r_{i j}$, less than a cutoff distance, $r_{c}=2.5 \mathrm{~nm}$. These quantities were obtained using the VOTCA [33] package and molecular orbital information calculated with GAUSSIAN [52].

Following many other models of charge transport in OSCs [12], the change in the Gibbs free energy $\Delta G_{i j}$ between pairs of molecules comes from electrostatic interactions between the charges in an applied bias. In a departure from standard practice, we solved the discretized Poisson's equation with a cloud-in-cell method to allow for the long-range nature of the Coulomb interactions [53],

$$
\bar{\phi}=-\nabla^{2} \frac{\bar{\rho}}{\epsilon},
$$

where $\bar{\phi}$ is the discretized electric potential and $\epsilon=\epsilon_{0} \epsilon_{r}$ is the permittivity of free space multiplied by the material's dielectric constant, with appropriate boundary conditions describing the applied bias. A coarse-grained charge density, $\bar{\rho}$, is defined on a grid, projecting the charges within each voxel onto the eight voxel vertices before solving Eq. (9). Each hopping site's electrostatic potential then comes from mapping $\bar{\phi}$ onto that site. The charge-density distribution and potential profile is recalculated after every KMC event.

The first reaction method (FRM) was used to select the next event to be performed in the KMC simulation [54]. In brief, the FRM method requires us to calculate the rate $\left(v_{k}\right)$ of every possible event in the system, and then for each event draw a waiting time $\left(t_{w}^{(k)}\right)$ from a Poissonian distribution parametrized by $v_{k}$. At each KMC step, we perform the event with the shortest waiting time. We note that for the given set of $M$ events with rates $v_{1}, \ldots, v_{M}$ and waiting times $t_{w}^{(1)}, \ldots, t_{w}^{(M)}$, the probability that the $k$ th event is performed is given by
$\mathrm{P}\left(t_{w}^{(k)}=\min \left[t_{w}^{(1)}, \ldots, t_{w}^{(M)}\right]\right)$, which is equivalent to

$$
\mathrm{P}\left(k \mid v_{1}, \ldots, v_{M}\right)=v_{k} / \sum_{i} v_{i} .
$$

We ran six simulations with different random number seeds for each morphology. The simulations were initiated with no charge carriers present in the cell. As the simulation ran, injection of charge carriers took place at the top electrode and extraction at the bottom electrode. Injection was treated as a two-step process in which the probability of a carrier being on a random hopping site adjacent to the electrode is multiplied by a Marcus hopping rate from the selected site to a site in the bulk of the device. The probability of being on an adjacent hopping site is calculated as $p=\min \left[1, e^{-\frac{\delta E}{k_{B} T}}\right]$, where $\delta E$ is the difference in carrier energy between the Fermi level of the electrode and the adjacent hopping site.

Once the dynamics reached steady state with respect to charge injection and extraction, measurements of the meansquare displacement, $\left\langle r^{2}(\tau)\right\rangle$, were taken at intervals of $\Delta t=$ $5 \times 10^{3} \mathrm{ps}$. If $\Delta t$ is too short, $\left\langle r^{2}(\tau)\right\rangle$ is dominated by charge transfer back and forth within a strongly coupled pair, in a rattling motion. Our choice of $\Delta t$ ensured that we measure charge motion that contributes to charge flow across the device. To measure the charge-carrier motion, we calculated the mean-square distance displacement of free charge carriers as a function of time $\tau$,

$$
\left\langle r^{2}(\tau)\right\rangle=\sum_{j=0}^{M} \frac{1}{N} \sum_{i=0}^{N}\left[\mathbf{r}_{i}\left(t_{j}+\Delta t\right)-\mathbf{r}_{i}\left(t_{j}\right)\right]^{2},
$$

where $M$ is the number of time steps. The maximum simulation time $\tau_{\max }$ was set at $10^{8} \mathrm{ps}$. Continuous measurements, such as the mean-squared displacement, were averaged over all KMC trajectories. The mobility was measured using

$$
\mu=\frac{D}{k_{B} T}, D=\operatorname{Lim}_{\tau \rightarrow \infty} \frac{\left\langle r^{2}(\tau)\right\rangle}{\tau},
$$

where $k_{B}$ is the Boltzmann constant.

\section{Network analysis}

To investigate the role of a given pair of molecules in charge transport, as compared to properties of the entire system, we need to use a description that treats pairs, rather than individual molecules, as the simplest individual object. To achieve this, we used tools from graph theory [43] that explicitly consider the connections between objects as entities in their own right. We mapped the transfer integrals onto a transportation network, called a graph, that consists of nodes linked by edges; an example is illustrated in Fig. 2. In this case, nodes are the HOTT molecules and edges are links between a molecule and all other molecules within the transfer cutoff $r_{c}$.

To probe charge transport, we use the charge trajectories to find the elements of a traffic matrix $\mathbf{T}$, an order $N$ square matrix with rows and columns each linked to molecules $i=1$ to $N$. Its diagonal elements are zero. Its off-diagonal elements, $\mathbf{T}_{i j}$, are equal to the number of charges that hopped between site $i$ and site $j$, i.e., the traffic from site $i$ to $j$.

We also define the linked traffic, $\hat{\mathbf{T}}_{i j}$, of an edge as the average traffic of the set of neighboring edges in a given 


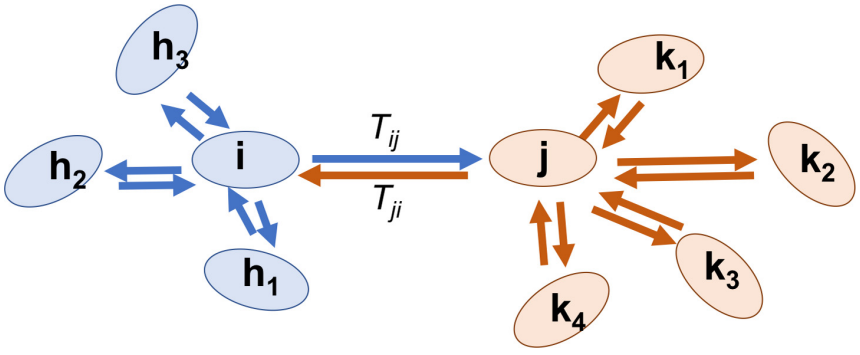

FIG. 2. Schematic illustration of the network. Nodes are the molecules shown as ellipses. For each pair of molecules separated by less than $r_{c}$ there are two edges whose directions are shown by the arrows joining the nodes. The node labels show how the linked traffic $\hat{T}_{i j}$ is calculated from Eq. (13).

direction. For an edge from $i$ to $j$, this set includes all edges that end at $i$ or start from $j$, excluding the reverse edge from $j$ to $i$. We can write $\hat{\mathbf{T}}_{i j}$,

$$
\hat{\mathbf{T}}_{i j}=\frac{1}{2}\left[\frac{\left(\sum_{h} \mathbf{T}_{h i}-\mathbf{T}_{j i}\right)}{M_{i}-1}+\frac{\left(\sum_{k} \mathbf{T}_{j k}-\mathbf{T}_{j i}\right)}{M_{j}-1}\right],
$$

where $M_{i}\left(M_{j}\right)$ is the number of neighboring nodes to node $i(j)$, and $h(k)$ is an index over this set. The sums are over neighboring edges, so the first (second) sum on the right-hand side is over all edges that begin at site $i(j)$ and end at site $j(i)$. Given that the traffic $\mathbf{T}_{i j}$ is an extensive measurement in time, all traffic measurements are made for the same simulation time $\tau_{\max }$. There is a net flux,

$$
\overrightarrow{\mathbf{F}}_{i j}=\left(\mathbf{T}_{i j}-\mathbf{T}_{j i}\right) \vec{e}_{i j} / \tau_{\max },
$$

where $\vec{e}_{i j}$ is the unit vector linking sites $i$ and $j$. Note that the flux matrix is skew-symmetric.

\section{RESULTS}

We simulated two morphologies of HOTT molecules at two different temperatures, 9011 molecules at $280 \mathrm{~K}$ and 8968 molecules at $400 \mathrm{~K}$, with the CG MD method described in Sec. II A. We used these two systems to investigate the effects of structural ordering on the distribution of charge-transfer integrals $J_{i j}$ as well as the long-range transport properties of the systems. Finally, we use the microscopic information embedded in the morphologies and KMC simulation to explain the trends in both short- and long-range charge transport.

The planar shape of the benzene rings allows molecules to form a $\pi-\pi$ stacking arrangement with little steric hindrance. Figure 3 shows the morphologies of the HOTT systems generated by back-mapping the coarse-grained morphologies into atomic coordinates. At the lower temperature, there is a clear long-range ordering of the molecules into columns. This ordering is destroyed at higher temperatures with no clear structure visible.

The peaks in Fig. 4 correspond to separations in a column of HOTT molecules with $r_{\|}$(Fig. 1) parallel to the column axis, as well as regular separations between columns in the radial plane. In the disordered phase, there is no ordering beyond first neighbors along $r_{\|}$and a broad correlation around $2.0 \mathrm{~nm}$.

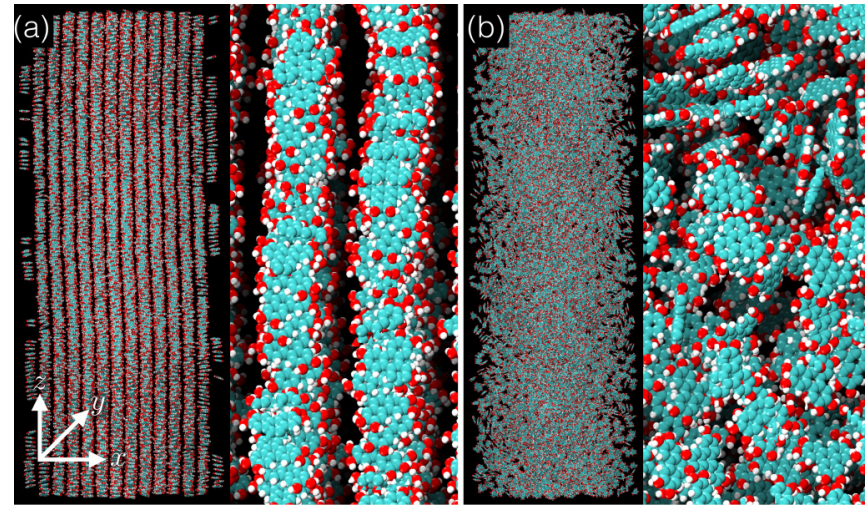

FIG. 3. HOTT morphologies after back-mapping to atomic coordinates. (a) Disordered morphology generated at $T=280 \mathrm{~K}$, system size is $15.67 \times 15.67 \times 52.85 \mathrm{~nm}$. (b) Columnar morphology generated at $T=400 \mathrm{~K}$, system size is $16.32 \times 16.32 \times 65.36 \mathrm{~nm}$.

Figure 5 shows the square of the transfer integrals between pairs of molecules, needed for the hopping rates in Eq. (8), against the spatial separation,

$$
\Delta \vec{r}=\Delta x \vec{e}_{x}+\Delta y \vec{e}_{y}+\Delta z \vec{e}_{z},
$$

using axes shown in Fig. 3. The distribution of $J^{2}$ in the columnar phase shows spatial ordering of the transfer integrals in all three dimensions. Although the $\Delta x$ and $\Delta y$ distributions are similar for Figs. 5(a) and 5(b) apart from where $J^{2} \approx$ $1 \times 10^{-12}$, the $\Delta z$ distribution is markedly different. In the $z$ direction, there are islands with large values of $J^{2}$ from coupling to the nearest and second-nearest molecules in the same column. Higher-order neighbors in the same column follow the same trend, although $J^{2}$ are of the same magnitude as for intercolumn transfer.

All possible hops are affected in the same way by an increase in temperature through Eq. (8), so if there is any favored set of hops (e.g., in columns), they would be favored at all temperatures. Thus the reason any particular coupling is more or less favored is not due to a global parameter such as

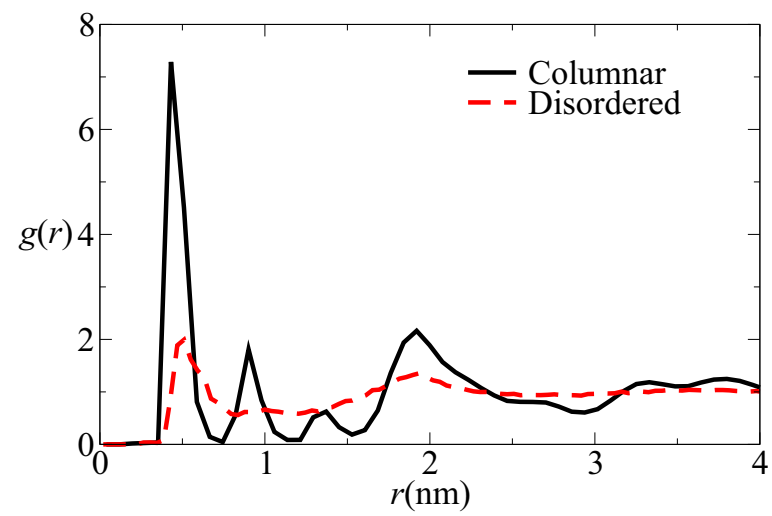

FIG. 4. Pair correlation function $g$ vs molecular separation $r(\mathrm{~nm})$ or HOTT molecules in columnar and disordered phases. For the former, the first peak is at $0.43 \mathrm{~nm}$ and represents the intracolumn vertical separation, the second peak is at $0.904 \mathrm{~nm}$, while the first minimum is at $0.74 \mathrm{~nm}$. The first peak in the disordered phase is at $0.515 \mathrm{~nm}$ 
(a)

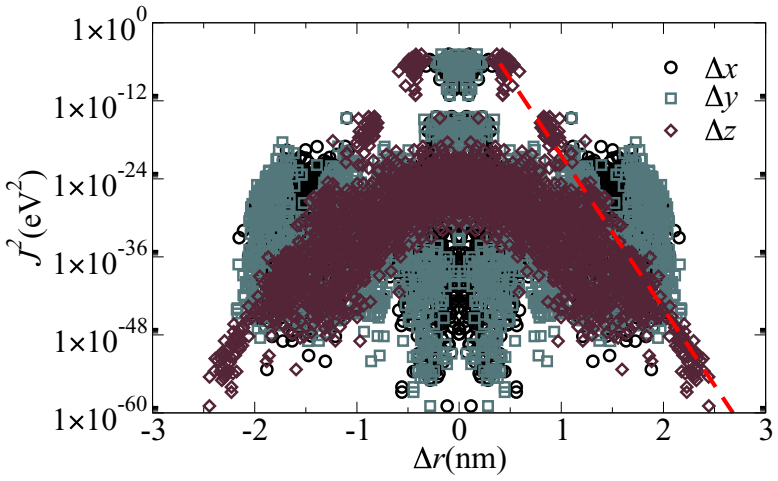

(b)

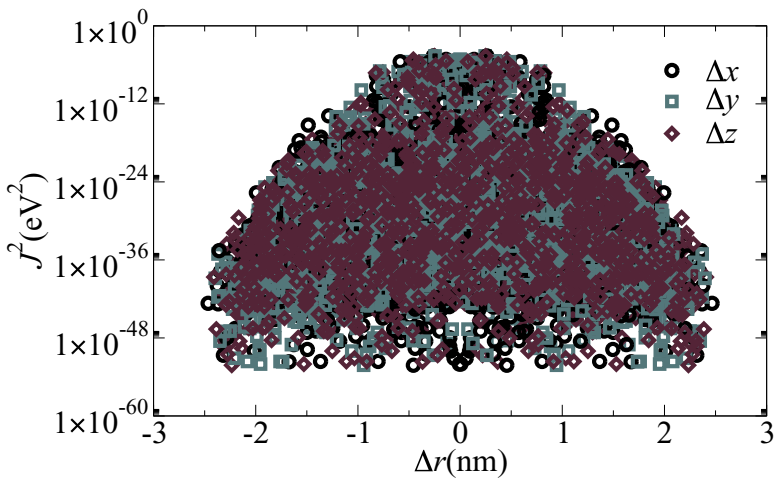

FIG. 5. The distribution of hole transfer integrals $J_{i j}^{2}$ between pairs of molecules with respect to the Cartesian components of the pair separation, according to $r_{i j}^{2}=\Delta x_{i j}^{2}+\Delta y_{i j}^{2}+\Delta z_{i j}^{2}$ (nm). (a) Columnar phase. The dashed red line illustrates the variation of $J^{2}$ when moving along a column. (b) Disordered phase.

temperature, but rather the local morphology. The distribution of smaller $J^{2}$ values that correspond to intercolumn hopping in the $z$ direction in the columnar phase is broad and continuous in both phases, falling off exponentially with $\Delta z$, shown by the dashed line in Fig. 5(a). Comparing the value of $\Delta z$ at which $J^{2}$ is large in Fig. 5(a) to the molecular dimensions given in Fig. 1, the most significant charge-transfer path corresponds to hopping between molecules separated by roughly $2 r_{\|}$ directly above or below one another.

$J^{2}$ variations in the $x-y$ plane shown in Fig. 5(a) distinguish between intra- and intercolumn hopping. The clustering of $\Delta x$ and $\Delta y$ around $\Delta r=0$ and $\Delta r=0.4 \mathrm{~nm}$ shows that the largest transfer integrals correspond to charge transfer within the same column. Intercolumn transfer occurs at $\Delta x \approx 1.9 \mathrm{~nm}$. Given that the column separation distances are much larger than $r_{c}$, there are few transfer integrals between molecules beyond nearest-neighbor columns. Figure 5(b) shows that the distribution of $J^{2}$ at $T=400 \mathrm{~K}$ is isotropic, as would be expected for a disordered morphology, and decays exponentially with $\Delta r$. The range of $J^{2}$ values is comparable to the ordered system, suggesting that the origin of large $J^{2}$ is the same at both temperatures: a pair of molecules arranged in a $\pi$-stacking configuration.

Looking at Figs. 4 and 5, we can see that although the nearest-neighbor separation is similar in both the columnar and isotropic phases, the distribution of pair separations in Cartesian directions and the distribution of transfer integrals

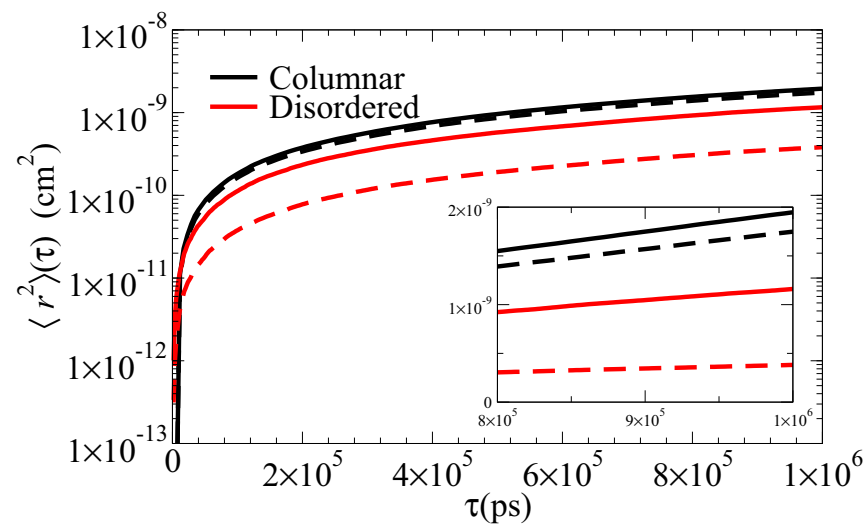

FIG. 6. Charge mean-squared displacement $\left\langle r_{z}^{2}\right\rangle$ (solid lines) and $\left\langle z_{z}^{2}\right\rangle$ (dotted lines) vs simulation time $\tau$ (ps) for columnar and disordered phases. Inset: a subsample of the same data on a linear scale.

are very different. One could not use a single lattice model to recreate both of these systems: the lattice would have to be a fine cubic mesh in the isotropic phase, while it would be a coarser stacked hexagonal lattice in the ordered phase. Even with different shaped lattices, one would need to describe the anisotropic coupling between molecules accurately. We stress that our model can describe all phases of a system accurately, and we will show below how the same methodology can be applied to both ordered and disordered systems.

A useful measure of charge transport is the mean-square distance traveled in a given time. Figure 6 shows that in the columnar phase, the ratio $\left\langle z^{2}\right\rangle /\left\langle r^{2}\right\rangle$ is nearly constant at 0.9 and motion along columns is strongly favored. By comparison, the charge transport in the disordered phase is isotropic, with transport parallel to the columns contributing a third of the total movement. That the motion is isotropic despite an applied voltage of $2.0 \mathrm{~V}$ shows that the change in energy due to movement parallel to the field is small compared to the intrinsic disorder and transfer integrals. In the columnar phase, the mobility $\mu_{z}=4.782 \times 10^{-6}\left(\mathrm{~cm}^{2} \mathrm{~V}^{-1} \mathrm{~s}^{-1}\right)$, and in the disordered phase its value is $\mu_{z}=7.938 \times 10^{-7}\left(\mathrm{~cm}^{2} \mathrm{~V}^{-1} \mathrm{~s}^{-1}\right)$ (see the supplemental material for further details).

The highly directional nature of the MSD in the columnar system suggests filamentary transport. It is known that one-dimensional transport depends upon the system size and eventually fails as a single defect severs the chain. Even if the transport chain is not severed, the link with the lowest rate will still be the rate-determining step with respect to long-range transport. To investigate this effect, we modified the hopping networks to halve the systems' effective height and changed the voltage to keep the field consistent. This should increase the observed mobility as the number of weak links should be reduced. The ratio of the halved system mobility to the original mobility, $\kappa=\mu_{z}^{\prime} / \mu_{z}$, is a measure of both the extent of filamentary transport and the density of weak connections in the filaments. In the columnar system, $\kappa=1.420$, while in the amorphous system, $\kappa=1.019$. These measurements reinforce the quantitative difference in the transport mechanisms: the columnar system is reducible to a set of separate conducting filaments, while the amorphous system is truly isotropic. 


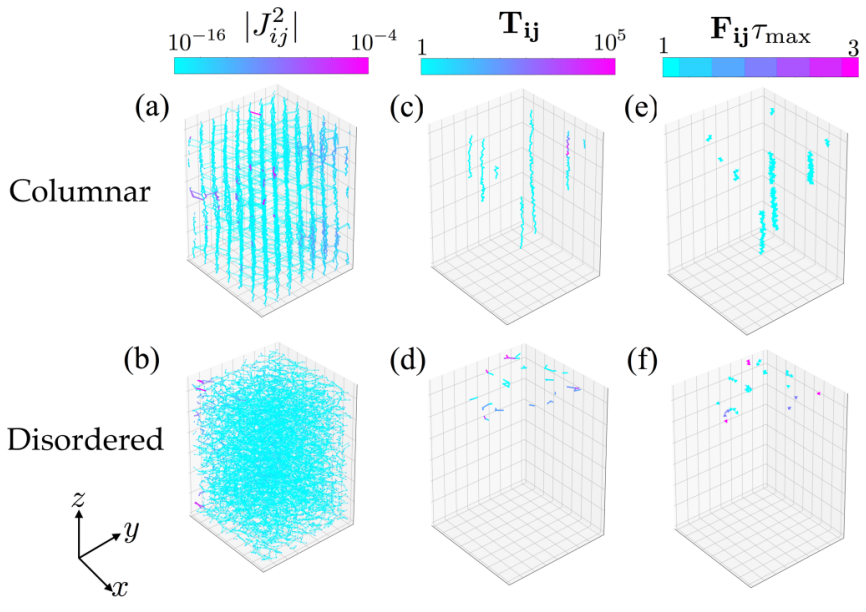

FIG. 7. Simulation cells where $0 \leqslant x \leqslant x_{\max }, 0 \leqslant y \leqslant y_{\max }, 0 \leqslant$ $z \leqslant z_{\max }$, and $x_{\max }, y_{\max }, z_{\max }$ are given in the caption to Fig. 3. (a) and (b) Edges with transfer integrals $\left|J_{i j}\right|^{2}>10^{-6} \mathrm{eV}$ shown as arrows; (c) and (d) traffic $\mathbf{T}$ between pairs (lines); (e) and (f) the extensive flux $\mathbf{F} \tau_{\max }$ integrated over $\tau_{\max }$ in columnar and disordered phases. The grids are to guide the reader's eye.

Our large system size allows us to look at spatial variations in charge transport. Figure 7(a) shows that in the columnar phase, the highest charge-transfer rates are between sites in the same column, while transfers between columns are typically much weaker, consistent with Fig. 5. The dark blue regions within columns indicate continuous chains of transfers. In the disordered system, Fig. 7(b) shows there is no spatial ordering of strongly coupled molecule pairs. Comparing Figs. 7(a) and 7(b) does not explain the difference in transport properties between the columnar and disordered systems, nor the extent of rattling motion. For both phases, we can see from Figs. 7(c) and 7(d) that very few pairs of molecules exchange free charge carriers. In the columnar phase, there is clear spatial ordering of charge hops along columns. In the disordered phase, even fewer pairs of molecules exchange carriers, and those that do are clustered in small groups of three or four hopping sites, although measures of transport should be evenly spread across the film.

Figures 7(c) and 7(d) show the number of hops along edges as colored arrows; edges where there are no hops are omitted. Dark blue edges that carry a noticeable level of traffic are clustered in small chains. The pink edges that carry exceptionally high traffic are isolated and have no obvious spatial distribution. From the applied color scale, we can see that the maximum observed numbers of hops in an edge are comparable in the two morphologies, although the number of edges that carry a noticeable level of traffic is smaller in the disordered system than the ordered system.

In graph theory, the disconnected clusters in Figs. 7(c) and 7 (d) are called subgraphs. Using $\mathbf{T}_{i j}$, we identified the number of sites in each subgraph as well as their length, i.e., the maximum extent of the subgraph parallel to the $z$ axis in Fig. 8 . In the ordered system, the clusters are filamentary. Their length scales linearly with the number of sites that are stacked in a column with a fixed pair separation of $6.5 \mathrm{~nm}$. Intercolumn connections in the $x-y$ plane do not play a role in charge

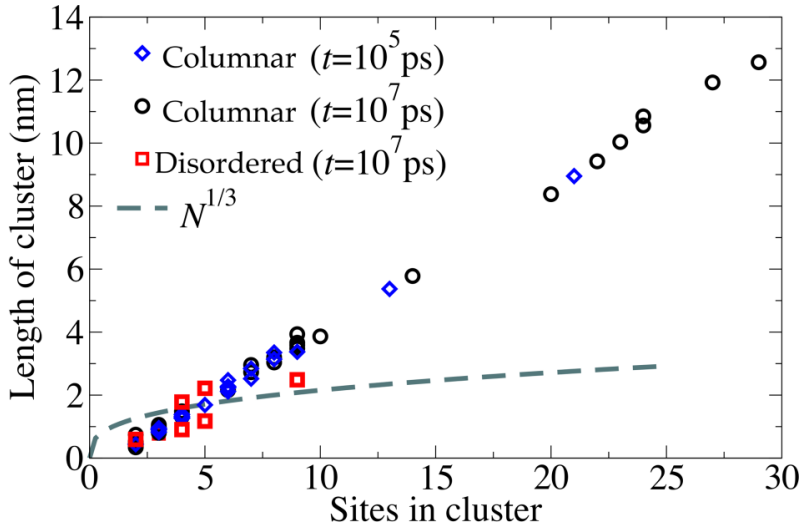

FIG. 8. Cluster size vs cluster length along the $z$ axis (the direction of an applied bias of $2 \mathrm{~V}$ ) for the columnar phase at simulation times of $10^{5}$ (blue diamonds) and $10^{7} \mathrm{ps}$ (circles) and for the disordered phase at $10^{7} \mathrm{ps}$ (red squares).

transport. In the disordered morphology, the cluster size scales as $N^{\frac{1}{3}}$ as can be seen in the figure. For a smaller simulation cell, the charge-transport length would be directly comparable to the percolation length, and there may only appear to be one or two favored pathways. The ability to simulate a large system means we can study the density and growth of these pathways.

It is known that one-dimensional transport will eventually fail as only a single fault will completely disrupt the entire transport network. In the system sizes presented, the columnar ordering percolates the system, thus we cannot describe the effect of dislocations to the charge transport. However, the study of the amorphous system shows that if two columnar regions are separated by a small amorphous region (a 1D crystal defect), transport will still occur, albeit slowly and isotropically. The regions between columns would effectively transport charge an order of magnitude more slowly than the columns; they would be the limiting factor to the overall performance of the device.

Figure 9 shows that the columnar phase exhibits a bimodal distribution with very few values of $J_{i j}$ around $10^{-6} \mathrm{eV}$, consistent with Fig. 5(a). This minimum corresponds to the first minimum in $g(r)$ at $r=0.74 \mathrm{~nm}$. At this molecular separation, there are very few molecule pairs that have the correct alignment for $J_{i j}$ to exceed its cutoff value (Fig. 4). The columnar morphology has more edges with large $J_{i j}$ than the disordered morphology, although the maximum observed $J_{i j}$ are comparable. These large $J_{i j}$ occur between particles that are at closest approach, equivalent to neighboring molecules that are parallel to the $x-y$ plane. The large number of connected particle pairs explains the faster charge transport shown in Fig. 6. In the disordered system, the distribution of particle separations is smoother and thus the distribution of $J_{i j}$ is smoother, too. As the strength of the coupling decreases with increasing pair separation, the two distributions have mirror symmetry. The dashed line in Fig. 9 shows that for the distribution of $J_{i j}$ over a smaller range of separations, the larger peak corresponds to the shoulder in the distribution of pair separations (see the figure inset); the shoulder in $P\left(J_{i j}\right)$ also has a counterpart in the separation distribution. Averaged over a large number of configurations, we would expect that 


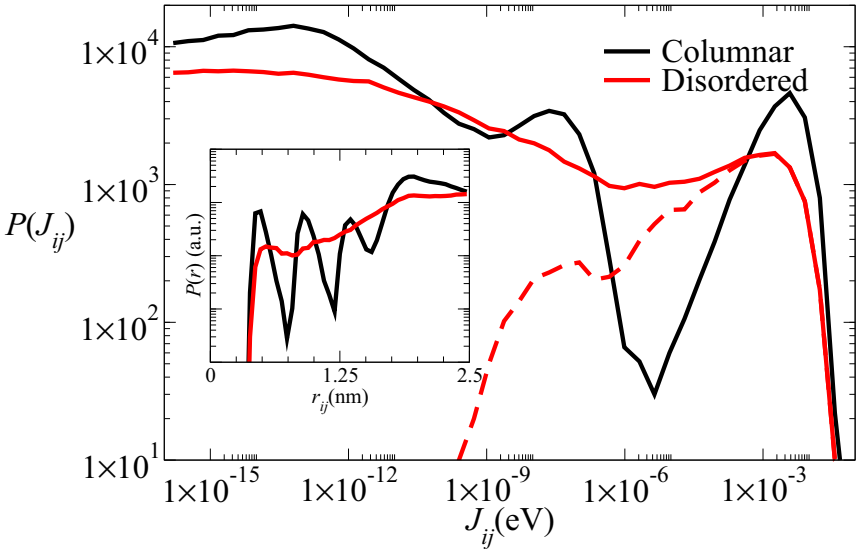

FIG. 9. Distribution of $J_{i j}$ in the columnar and disordered phases. For the former there is a clear bimodal distribution due to the high spatial and orientational ordering of the molecules. For the latter, the distribution is flatter. The solid line is the distribution taken over all molecule pairs up to $2.5 \mathrm{~nm}$ apart; the dashed line is for pairs up to 1 $\mathrm{nm}$. Inset: the distribution of pair separations in the two phases.

the distribution of $J_{i j}$ at a fixed value of $r_{i j}$, or a range small enough to only include nearest-neighbor pairs, would become Gaussian due to the central limit theorem.

Even for the ordered morphology, the transport is poor. To get an estimate of how few edges contribute to charge transport, we can compare $N_{e}^{\prime}$, the number of edges where $\mathbf{T}_{i j}>0$, to the total number of edges $N_{e}$. At a bias of $2 \mathrm{~V}$ in the columnar phase, the ratio $N_{e}^{\prime} / N_{e}=1.17 \times 10^{-3}$ is an indicator of the variability in transport behavior between molecules. In the disordered system, the ratio is an order of magnitude smaller, and carrier hops are more localized along fewer edges. This suggests that the distribution of transfer rates is more sparse. To quantify the degree of rattling motion, we calculate $\tilde{N}_{\text {hops }}=\frac{1}{2} \sum\left|\mathbf{F}_{i j}\right|$, the number of carrier hops that contribute to a flux. The ratio of $\tilde{N}_{\text {hops }}$ to the total number of recorded hops is small, of the order $10^{-4}$ in the columnar phase and an order of magnitude smaller in the disordered phase, suggesting that most carrier hops represent rattling motion. More precise values of these quantities are included in the supplemental material [56].

The effect of the transfer integral distribution in the columnar phase is that hops between columns are very unlikely despite the change in Gibbs free energy, $\Delta G_{i j}$, being roughly equal for intra- and intercolumn edges. Although the transfer integrals limit charge motion to the $z$ axis, they do not dictate whether charges hop up or down columns, hence any net transport must be driven by energetic considerations. This means that a requirement for long-range transport is for the set of edges with $\Delta G_{i j} \approx-\lambda$ to be asymmetrically distributed around $\Delta z=0$. Changes in site energies due to energetic disorder are in general much larger than the electrostatic energy changes when moving between nearest neighbors. The weakness of the asymmetry is critical to understanding why the number of edges that contribute to flux is so small. We can visualize this argument from Fig. 1 of the supplemental material (SM) [56].

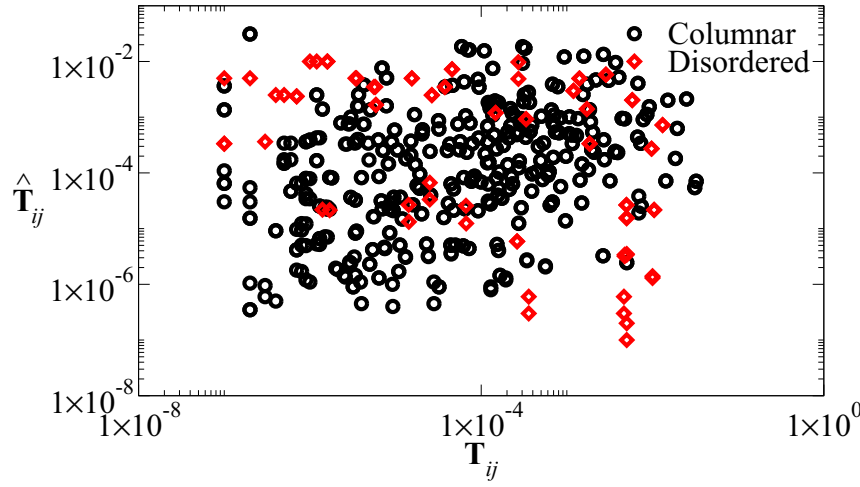

FIG. 10. The traffic between two molecular sites vs the linked traffic of that pair as defined in Eq. (13). Both measures of the traffic are more tightly distributed in the ordered system.

Due to the low asymmetry of $\Delta G_{i j}$ with respect to $\Delta z_{i j}$, few edges contribute to the carrier flux matrix $\mathbf{F}$. The closer $-\lambda$ is to the bottom edge of the distribution of $\Delta G_{i j}$, or the larger the optimal transfer integral separation, the weaker the applied bias needs to be. Figure 10 shows the traffic along a given edge, $\mathbf{T}_{i j}$, plotted against the linked traffic of the edge, $\hat{\mathbf{T}}_{i j}$. There is little correlation between the traffic of an edge and the average traffic of its neighbors. However, in the ordered morphology the distribution of both traffic measures is narrower and closer to equality between the two measures. This result suggests that in the ordered system, the flow along edges is more uniform and the transport environment around an edge is similar to that of the edge concerned. We can relate the strong correlation between structural ordering and electronic coupling to the dynamics of long-range charge transport: structural ordering means less variation in local structural environment (see Fig. 4) and corresponds to $\pi$-stacking in this system. The stacking results in a relatively narrow distribution of transfer integrals (see Fig. 9), affecting the dynamic behavior, and the traffic along edges is distributed relatively narrowly around equality between $\left\langle\mathbf{T}_{i j}\right\rangle=\mathbf{T}_{i j}$.

The edges that host most charge-hopping events occur for $\Delta G_{i j} \approx-\lambda$. Outside this range, the rate of a hop is at least $10^{7}$ times smaller. Comparing two hopping events with such large differences in rates, we can see that when $v_{1} \gg v_{2}$, using Eq. (10) the probability that the waiting times satisfy $t_{w}^{(1)}<$ $t_{w}^{(2)}=v_{1} /\left(v_{1}+v_{2}\right) \approx 1$. Recall that in the FRM we perform the event with the smallest waiting time at each iteration. Thus only hopping events with rates close to the maximum rate will occur, and so only those events with $\Delta G_{i j}$ close to $-\lambda$ are relevant for transport. This limitation of charge transport to a narrow range of $\Delta G_{i j}$ explains why so few edges carry any traffic. If an OSC had a reorganization energy close to the typical difference in energy between pairs, determined by the disorder in HOMO/LUMO energies, then many more edges would be involved in transport.

If the transfer integral dominates in Eq. (8), then charge hopping is not spatially confined, and despite the differences in $\Delta G_{i j}$, many edges are equally likely to carry traffic. In practice, the exponential factor dominates, so despite many edges having similar transfer integrals, those with $\Delta G_{i j}$ closest to $-\lambda$ carry almost all the traffic. We suggest that to identify 


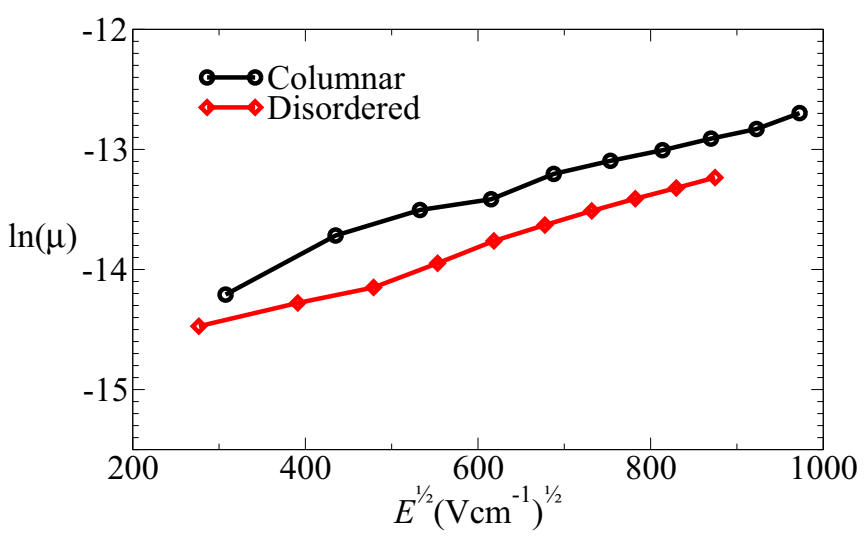

FIG. 11. Poole-Frenkel mobility-field plot for columnar and disordered phases. The mobility increases with increasing applied field in both systems, although the response is much stronger in the columnar system. The weak dependence upon the applied field in the amorphous system is consistent with Figs. 5 and 6; the field is more effective when aligned to the coupling topology.

high transfer rate edges, it is more useful to consider that high transfer integrals select from the set of edges with $\Delta G_{i j} \approx-\lambda$, than favorable values of $\Delta G_{i j}$ select from the set of all edges with high $J^{2}$. This selective pressure explains why so few edges in the $400 \mathrm{~K}$ morphology carry traffic despite the isotropic nature of the transfer integrals. For predictive power, one must know the distribution of $\Delta G_{i j}$, or at least $\Delta G_{i j}$ in the absence of charges, in the system as well as $\left\{J_{i j}\right\}$ to identify favored traffic directions.

The disordered morphology, which exhibits slower longrange charge transport, contains molecule pairs that exchange $\sim 10^{4}$ holes during the simulation. Figures 7(b) and 7(d) show that high traffic edges are isolated and the connected edges are not strong, either with respect to the transfer integral or the observed traffic. This lack of connectedness leads to a relatively small contribution to transport despite high coupling and traffic.

The difference in energy between two sites is composed of the inherent energetic disorder and electrostatic contributions. As the applied field is increased, it becomes relatively large compared to the disorder and $\Delta G_{i j}$ becomes correlated with $\Delta z_{i j}$. The gradient of Fig. 11 is positive since the application of a field breaks the symmetry of $\Delta G_{i j}$ about $\Delta z=0$. The population of edges with $\Delta z<0$ moves closer to $\Delta G_{i j} \approx=$ $\lambda$ while those with $\Delta z_{i j}>0$ move farther away. A drift component parallel to the field is therefore superimposed on the the random motion of charges. Beyond an optimum bias, the typical hopping rate would decrease due to the inverted region of the Marcus equation dominating when $\Delta G_{i j} \ll=\lambda$. In the ordered system, the most highly connected edges are already parallel to the field so the effect is amplified, while in the disordered system an increasing field will only favor those edges that are aligned correctly. No electric field dependence was observed by Kwiatkowski et al. [14], suggesting that the origin of such a dependence is more subtle than simply the presence of energetic disorder. We suggest that the topology of the electronic transport network, defined by transfer integrals, plays a role in the electric-field dependence of the mobility.

\section{CONCLUSIONS}

We compared the charge-transport properties of an organic semiconductor discotic system in two distinct structural phases using explicitly calculated electronic transfer integrals and orbital energies in a kinetic Monte Carlo simulation. The ordered columnar phase exhibits much higher charge-carrier mobility parallel to the column axis, while transport between columns is very rare. Intercolumn transport is restricted by weak transfer integrals, and the change in free energy both along and between columns is roughly equal due to energetic disorder.

Discrete charge transport via hopping mechanisms maps well to graph theory methods and discussion of edges and nodes. Metrics that can describe paths of arbitrary length across the system and measures of robustness help identify good charge-transport criteria. If efficient charge transport is more reliant on the collective behavior of a series of hops, on pathways rather than pairs, then graph theory is a natural expression. In the future, it would be interesting to see if this approach could be used to define which sites in the system contribute to charge flux and which can be described as dynamic traps. These sites are likely to be important for light emission and recombination in optoelectronic devices.

Although the energetic term dominates the Marcus rate equation, it is still important to calculate the transfer integrals explicitly. If we only consider hopping due to energetic terms, we would expect to see as many hops between columns as along columns, although symmetry would lead to $\langle\Delta x\rangle=\langle\Delta y\rangle=0$. Instead we see no hops between columns at all, due to the much weaker electronic coupling between columns, as shown in Fig. 5. By calculating the transfer integrals between each pair of molecules, we reproduced the strong coupling caused by coplanar aromatic rings. However, the distribution of transfer integrals does not promote net charge transport along columns either; the distribution is symmetric with respect to $\Delta z$.

The distribution of $\Delta G_{i j}$ is weakly asymmetric with respect to $\Delta z$-it is this asymmetry that leads to net flow of charge carriers. The asymmetry caused by the change in energy due to the applied field between molecules $i$ and $j$ is $q \Delta \phi=$ $-q \vec{F} \cdot \vec{r}_{i j}$, where $\vec{F}$ is the local field and $\vec{r}_{i j}$ is the displacement between the molecules. The exponential decay of the transfer integrals with separation means that increasing the asymmetry by increasing the nearest-neighbor separations is not possible, but increasing the applied field does increase the mobility. The largest transfer integrals inside columns are linked to an intermolecular separation $d=0.4 \mathrm{~nm}$ parallel to the field with an applied bias of $2 \mathrm{~V}\left|q \vec{F}\left\langle\vec{r}_{i j}\right\rangle\right|=0.585 k_{B} T$. Comparing the thermal energy to the driving field energy, we can see why charge flux is so small and why rattling motion dominates at low applied fields.

We used the KMC trajectories to define a measure of locally correlated charge flow and thus to identify edges (directed molecular pairs as shown in Fig. 2) that carry high traffic but contribute little to flow, and conversely edges that carry relatively little traffic but are well connected in terms of flow. More important to particle flux is the connectedness of edges with large transfer integrals to create a pathway. We see that the disordered system has the same range of transfer integrals compared to the ordered system, yet the charge transport is slower. The lack of spatial correlations between edges with 
good transport properties means that it is unlikely that carriers will reach good edges, and if they do they are unlikely to move far away. This resolved the paradox that the disordered phase contains edges that carry an order of magnitude more traffic than the columnar phase, yet it performs worse with respect to overall charge transport. To achieve efficient charge transport over device length scales, it is necessary to have a continuous chain of hops, all with reasonable electronic coupling. Furthermore, the weighting of any single edge is a poor indicator of how effective that edge is in terms of long-range transport. There is little correlation between edge traffic and the average traffic of its neighbors. Assuming that the structural order of a system is compatible with high electronic coupling, as in $\pi$-stacked systems, we can directly correlate structural ordering to the motion of charges.
The hop with the slowest rate is the most critical hop with respect to the overall transport time along the chain, both in terms of being the slowest hop forward but also increasing the relative probability of a reverse hop back along the chain. Reducing the variance in edge weights means that the probability of one pathological molecule pair in a chain is reduced. This leads to improved charge transport across the entire chain. Isolated high-weight edges host high traffic without longer-range flow.

\section{ACKNOWLEDGMENTS}

The authors acknowledge funding from the European Union Horizon 2020 Research and Innovation Programme under Grant Agreement No. 646176.
[1] Nature Publishing Group, Editorial: Embracing the organics world, Nat. Mater. 12, 591 (2013).

[2] J.-L. Bredas, D. Beljonne, V. Coropceanu, and J. Cornil, Charge-transfer and energy-transfer processes in $\pi$-conjugated oligomers and polymers: A molecular picture, Chem. Rev. 104, 4971 (2004).

[3] V. Lemaur, M. Steel, D. Beljonne, J. L. Bredas, and J. Cornil, Photoinduced charge generation and recombination dynamics in model donor/acceptor pairs for organic solar cell applications: A full quantum-chemical treatment, J. Am. Chem. Soc. 127, 6077 (2005).

[4] D. Markovitsi, F. Rigaut, M. Mouallem, and J. Malthête, One-dimensional energy migration in crystalline and columnar liquid-crystalline phases of $2,3,6,7,10,11-$ hexa-n-hexyloxytriphenylene, Chem. Phys. Lett. 135, 236 (1987).

[5] N. Boden, R. J. Bushby, J. Clements, B. Movaghar, K. J. Donovan, and T. Kreouzis, Mechanism of charge-transport in discotic liquid-crystals, Phys. Rev. B 52, 13274 (1995).

[6] F. M. Mulder, J. Stride, S. J. Picken, P. Kouwer, M. P. de Haas, L. Siebbeles, and G. J. Kearley, Dynamics of a triphenylene discotic molecule, hat6, in the columnar and isotropic liquid phases, J. Am. Chem. Soc. 125, 3860 (2003).

[7] S. Sergeyev, W. Pisula, and Y. H. Geerts, Discotic liquid crystals: A new generation of organic semiconductors, Chem. Soc. Rev. 36, 1902 (2007).

[8] X. L. Feng, V. Marcon, W. Pisula, M. R. Hansen, J. Kirkpatrick, F. Grozema, D. Andrienko, K. Kremer, and K. Mullen, Towards high charge-carrier mobilities by rational design of the shape and periphery of discotics, Nat. Mater. 8, 421 (2009).

[9] M. Lamarra, L. Muccioli, S. Orlandi, and C. Zannoni, Temperature dependence of charge mobility in model discotic liquid crystals, Phys. Chem. Chem. Phys. 14, 5368 (2012).

[10] T. Wohrle, I. Wurzbach, J. Kirres, A. Kostidou, N. Kapernaum, J. Litterscheidt, J. C. Haenle, P. Staffeld, A. Baro, F. Giesselmann, and S. Laschat, Discotic liquid crystals, Chem. Rev. 116, 1139 (2016).

[11] C. Ruiz, U. K. Pandey, R. Termine, E. M. Garcia-Frutos, G. Lopez-Espejo, R. P. Ortiz, W. Huang, T. J. Marks, A. Facchetti, M. C. R. Delgado, A. Golemme, and B. Gomez-Lor, Mobility versus alignment of a semiconducting pi-extended discotic liquid-crystalline triindole, ACS Appl. Mater. Interf. 8, 26964 (2016).

[12] C. Groves, Simulating charge transport in organic semiconductors and devices: A review, Rep. Progr. Phys. 80, 026502 (2017).

[13] J. Nelson, J. J. Kwiatkowski, J. Kirkpatrick, and J. M. Frost, Modeling charge transport in organic photovoltaic materials, Acc. Chem. Res. 42, 1768 (2009).

[14] J. J. Kwiatkowski, J. Nelson, H. Li, J. L. Bredas, W. Wenzel, and C. Lennartz, Simulating charge transport in tris $(8$ hydroxyquinoline) aluminium $\left(\mathrm{alq}_{3}\right)$, Phys. Chem. Chem. Phys. 10, 1852 (2008).

[15] S. Tiwari and N. C. Greenham, Charge mobility measurement techniques in organic semiconductors, Opt. Quantum Electron. 41, 69 (2009).

[16] G. Gryn'ova, A. Nicolai, A. Prlj, P. Ollitrault, D. Andrienko, and C. Corminboeuf, Charge transport in highly ordered organic nanofibrils: Lessons from modelling, J. Mater. Chem. C 5, 350 (2017).

[17] A. Fischer, P. Pahner, B. Lüssem, K. Leo, R. Scholz, T. Koprucki, K. Gärtner, and A. Glitzky, Self-Heating, Bistability, and Thermal Switching in Organic Semiconductors, Phys. Rev. Lett. 110, 126601 (2013).

[18] M. Mesta, M. Carvelli, R. J. de Vries, H. van Eersel, J. J. M. van der Holst, M. Schober, M. Furno, B. Lüssem, K. Leo, P. Loebl, R. Coehoorn, and P. A. Bobbert, Molecular-scale simulation of electroluminescence in a multilayer white organic light-emitting diode, Nat. Mater. 12, 652 (2013).

[19] H. Bässler, Charge transport in disordered organic photoconductors a Monte Carlo simulation study, Phys. Status Solidi B 175, 15 (1993).

[20] W. F. Pasveer, J. Cottaar, C. Tanase, R. Coehoorn, P. A. Bobbert, P. W. M. Blom, D. M. de Leeuw, and M. A. J. Michels, Unified Description of Charge-Carrier Mobilities in Disordered Semiconducting Polymers, Phys. Rev. Lett. 94, 206601 (2005).

[21] B. I. Shklovskii, Hopping conduction in semiconductors subjected to a strong electric field, Sov. Phys. Semicond. 6, 1964 (1973).

[22] F. Jansson, S. D. Baranovskii, F. Gebhard, and R. Österbacka, Effective temperature for hopping transport in a Gaussian density of states, Phys. Rev. B 77, 195211 (2008). 
[23] J. O. Oelerich, A. V. Nenashev, A. V. Dvurechenskii, F. Gebhard, and S. D. Baranovskii, Field dependence of hopping mobility: Lattice models against spatial disorder, Phys. Rev. B 96, 195208 (2017).

[24] M. O'Neill and S. M. Kelly, Ordered materials for organic electronics and photonics, Adv. Mater. 23, 566 (2011).

[25] Y. Maeda, D. S. S. Rao, S. K. Prasad, S. Chandrasekhar, and S. Kumar, Phase behavior of the discotic mesogen 2,3,6,7,10,11hexahexylthiotriphenylene (hhtt) under hydrostatic pressure, Liq. Cryst. 28, 1679 (2001).

[26] N. Boden, R. J. Bushby, and A. N. Cammidge, Functionalisation of triphenylene based discotic liquid crystals, Mol. Cryst. Liq. Cryst. A 260, 307 (1995).

[27] S. Orlandi, L. Muccioli, M. Ricci, and C. Zannoni, Core charge distribution and self assembly of columnar phases: The case of triphenylenes and azatriphenylenes, Chem. Centr. J. 1, 15 (2007).

[28] A. M. van der Craats, J. M. Warman, M. P. de Haas, D. Adam, J. Simmerer, D. Haarer, and P. Schuhmacher, The mobility of charge carriers in all four phases of the columnar discotic material hexakis(hexylthio)triphenylene: Combined tof and prtrmc results, Adv. Mater. 8, 823 (1996).

[29] G. Cinacchi, R. Colle, and A. Tani, Atomistic molecular dynamics simulation of hexakis(pentyloxy)triphenylene: Structure and translational dynamics of its columnar state, J. Phys. Chem. B 108, 7969 (2004).

[30] J. M. Warman, M. P. de Haas, G. Dicker, F. C. Grozema, J. Piris, and M. G. Debije, Charge mobilities in organic semiconducting materials determined by pulse-radiolysis time-resolved microwave conductivity: $\pi$-bond-conjugated polymers versus $\pi-\pi$-stacked discotics, Chem. Mater. 16, 4600 (2004).

[31] M. A. Palenberg, R. J. Silbey, M. Malagoli, and J.-L. Bredas, Almost temperature independent charge carrier mobilities in liquid crystals, J. Chem. Phys. 112, 1541 (2000).

[32] M. O’Neill and S. M. Kelly, Liquid crystals for charge transport, luminescence, and photonics, Adv. Mater. 15, 1135 (2003).

[33] V. Rühle, A. Lukyanov, F. May, M. Schrader, T. Vehoff, J. Kirkpatrick, B. Baumeier, and D. Andrienko, Microscopic simulations of charge transport in disordered organic semiconductors, J. Chem. Theor. Comput. 7, 3335 (2011).

[34] P. Kordt, T. Speck, and D. Andrienko, Finite-size scaling of charge carrier mobility in disordered organic semiconductors, Phys. Rev. B 94, 014208 (2016).

[35] A. Massé, R. Coehoorn, and P. A. Bobbert, Universal Size Dependent Conductance Fluctuations in Disordered Organic Semiconductors, Phys. Rev. Lett. 113, 116604 (2014).

[36] M. F. Palermo, A. Pizzirusso, L. Muccioli, and C. Zannoni, An atomistic description of the nematic and smectic phases of 4n-octyl-4' cyanobiphenyl (8cb), J. Chem. Phys. 138, 204901 (2013).

[37] G. Tiberio, L. Muccioli, R. Berardi, and C. Zannoni, Towards in silico liquid crystals. Realistic transition temperatures and physical properties for n-cyanobiphenyls via molecular dynamics simulations, ChemPhysChem 10, 125 (2009).

[38] D. Beljonne, J. Cornil, L. Muccioli, C. Zannoni, J.-L. Brédas, and $\mathrm{F}$. Castet, Electronic processes at organic-organic interfaces: Insight from modeling and implications for opto-electronic devices, Chem. Mater. 23, 591 (2011).

[39] Y. Olivier, L. Muccioli, and C. Zannoni, Quinquephenyl: The simplest rigid-rod-like nematic liquid crystal, or is it? An atomistic simulation, ChemPhysChem 15, 1345 (2014).
[40] D. M. DeLongchamp, R. J. Kline, and A. Herzing, Nanoscale structure measurements for polymer-fullerene photovoltaics, Energy Environ. Sci. 5, 5980 (2012).

[41] D. Frenkel and B. Smit, Understanding Molecular Simulations: From Algorithms to Applications (Academic, San Diego, 2002).

[42] O. M. Roscioni and C. Zannoni, Molecular dynamics simulation and its applications to thin-film devices, in Unconventional Thin Film Photovoltaics, edited by E. Da Como, F. De Angelis, H. Snaith, and A. B. Walker (Royal Society of Chemistry, London, 2016), pp. 391-419.

[43] R. J. Wilson, Introduction to Graph Theory, Longman Scientific \& Technical (Longman, Harlow, Essex, 1985).

[44] N. E. Jackson, L. X. Chen, and M. A. Ratner, Charge transport network dynamics in molecular aggregates, Proc. Natl. Acad. Sci. (U.S.A.) 113, 8595 (2016).

[45] J. Cottaar, R. Coehoorn, and P. A. Bobbert, Scaling theory for percolative charge transport in molecular semiconductors: Correlated versus uncorrelated energetic disorder, Phys. Rev. B 85, 245205 (2012).

[46] M. Stamatakis and D. G. Vlachos, A graph-theoretical kinetic Monte Carlo framework for on-lattice chemical kinetics, J. Chem. Phys. 134, 214115 (2011).

[47] Y. Maeda, D. S. S. Rao, S. K. Prasad, S. Chandrasekhar, and S. Kumar, Phase behavior of the discotic mesogen 2,3,6,7,10,11hexahexyl thiotriphenylene (hhtt) under pressure, Mol. Cryst. Liq. Cryst. 397, 129 (2003)

[48] A. P. J. Emerson, G. R. Luckhurst, and S. G. Whatling, Computer-simulation studies of anisotropic systems xxiii. The Gay-Berne discogen, Mol. Phys. 82, 113 (1994).

[49] P. K. Watkins, A. B. Walker, and G. L. B. Verschoor, Dynamical Monte Carlo modelling of organic solar cells: The dependence of internal quantum efficiency on morphology, Nano Lett. 5, 1814 (2005).

[50] R. A. Marcus, On the theory of oxidation-reduction reactions involving electron transfer. I, J. Chem. Phys. 24, 966 (1956).

[51] P. Th. van Duijnen and M. Swart, Molecular and atomic polarizabilities: Thole's model revisited, J. Phys. Chem. A 102, 2399 (1998).

[52] M. J. Frisch, G. W. Trucks, H. B. Schlegel, M. E. Scuseria, M. A. Robb, J. R. Cheeseman, G. Scalmani, V. Barone, G. A. Petersson, H. Nakatsuji, X. Li, M. Caricato, A. Marenich, J. Bloino, B. G. Janesko, R. Gomperts, B. Mennucci, H. P. Hratchian, J. V. Ortiz, A. F. Izmaylov, J. L. Sonnenberg, D. Williams-Young, F. Ding, F. Lipparini, F. Egidi, J. Goings, B. Peng, A. Petrone, T. Henderson, D. Ranasinghe, V. G. Zakrzewski, J. Gao, N. Rega, G. Zheng, W. Liang, M. Hada, M. Ehara, K. Toyota, R. Fukuda, J. Hasegawa, M. Ishida, T. Nakajima, Y. Honda, O. Kitao, H. Nakai, T. Vreven, K. Throssell, J. A. Montgomery Jr., J. E. Peralta, F. Ogliaro, M. Bearpark, J. J. Heyd, E. Brothers, K. N. Kudin, V. N. Staroverov, T. Keith, R. Kobayashi, J. Normand, K. Raghavachari, A. Rendell, J. C. Burant, S. S. Iyengar, J. Tomasi, M. Cossi, J. M. Millam, M. Klene, C. Adamo, R. Cammi, J. W. Ochterski, R. L. Martin, K. Morokuma, O. Farkas, J. B. Foresman, and D. J. Fox, Gaussian 09 revision a.02 (Gaussian Inc., Wallingford, CT, 2009).

[53] R. W. Hockney and J. W. Eastwood, Computer Simulation Using Particles (Taylor \& Francis, Bristol, PA, 1988). 
[54] D. T. Gillespie, A general method for numerically simulating the stochastic time evolution of coupled chemical reactions, J. Comput. Phys. 22, 404 (1976).

[55] S. Marguet, D. Markovitsi, P. Millié, H. Sigal, and S. Kumar, Influence of disorder on electronic excited states: An experimental and numerical study of alkylthiotriphenylene columnar phases, J. Phys. Chem. B 102, 4697 (1998).
[56] See Supplemental Material at http://link.aps.org/supplemental/ 10.1103/PhysRevMaterials.2.064601 for more information regarding asymmetry in the distribution of the change in the Gibbs energy between sites, specifically with respect to the value of the reorganization energy. Also included in the supplemental information is a table of the measured rattling parameters. 